\section{Personalidad y competencias del psicoterapeuta de niños, niñas y adolescentes que han vivido experiencias de abuso sexual, revisión teórica ${ }^{5}$}

Laura Isaza Valencia

Ps. Doctoranda en Humanidades Universidad Pontificia Bolivarana Medellín, Colombia

Correo: laura.isazava@upb.edu.co

\section{Luz E. Ocampo Otálvaro}

PhD. Psicología Universidad Pontificia Bolivariana Medellín, Colombia

Recibido: 05/06/2015 Evaluado: $12 / 11 / 2015$ Aceptado: 12/11/2015

\title{
Resumen
}

Objetivo: comprender teóricamente la problemática del abuso sexual de niños, niñas y adolescentes, y la necesidad de identificar las características de personalidad y competencias del psicoterapeuta, debido a la influencia que tienen éstas en el establecimiento de la relación paciente- terapeuta. Método: Fue un estudio cualitativo de tipo documental descriptivo, se revisaron artículos teóricos y de investigación a partir del uso de 12 bases; se realizó una selección de las fuentes de información como criterio el año de publicación (2005-2013), se sistematizó la información y se crearon categorías de análisis que permitieron establecer y profundizar los factores asociados al tema del estudio. Resultados: Las categorías identificadas, analizadas y teorizadas, fueron: Estilo Personal del Terapeuta, como factor fundamental para el establecimiento de alianza en los procesos terapéuticos; Características de Personalidad del Terapeuta, componentes esenciales que intervienen en el establecimiento de la relación con el paciente; Abuso Sexual, problemática social que genera efectos físicos, psicológicos y sociales para la víctima y su familia; Terapia Infantil en el Abuso Sexual, proceso esencial para favorecer la superación del impacto negativo del abuso; y Terapia Familiar en el Abuso Sexual, proceso terapéutico centrado en el núcleo familiar con la red como estrategia integradora que permite hacer ruptura con las vivencias del abuso. Conclusiones: En esta revisión teórica se identificó cinco categorías de análisis esenciales para la comprensión de la problemática del abuso sexual de niños, niño y adolescente; el reconocimiento de la importancia de las características de personalidad y competencias del terapeuta en la atención de esta problemática, y la función preponderante de la empatía y alianza en la relación paciente- terapeuta.

Palabras clave

Abuso sexual, Alianza, Personalidad, Psicoterapia, Terapia.

5 Para Citar este artículo: Izasa, L., \& Ocampo, L. (2015). Personalidad del terapeuta de niños, niñas y adolescentes que han vivido experiencias de abuso sexual, revisión teórica. Informes Psicológicos, 15(2), 93-110. http://dx.doi. org/10.18566/infpsicv15n2a05 


\section{Personality and competencies of the psychotherapist for boys, girls and adolescents who have had sexual abuse experiences: theoretical review}

Abstract

Aim: To theoretically understand the problem of sexual abuse of boys, girls and adolescents, and the need to identify the personality traits and competencies of the psychotherapist, due to the influence that these may have in the establishment of the patient-therapist relationship. Method: It was a descriptive documentary-like qualitative study; theoretical and research articles were reviewed based on the use of 12 bases; a selection of the sources of information took place; as criterion, the year of publication 2005-2013; information was systematized and categories of analysis were created that enabled the establishment and deepening of the factors associated with the subject of study. Results: The categories identified, analyzed and theorized were: Personal Style of the Therapist, as a key factor for the establishment of partnership in the therapeutic processes; Personality Traits of the Therapist, essential components involved in the establishment of the relationship with the patient; Sexual Abuse, social problems that generate physical, psychological and social effects on the victim and their family; Child Therapy on Sexual Abuse, essential process to favour the overcoming of the negative impact of the abuse; and Family Therapy on Sexual Abuse, therapeutic process focused on the family with the network as an integrating strategy that allows to make a break with the experiences of abuse. Conclusions: In this theoretical review, we identified five categories of analysis which are essential to the understanding of the problem of sexual abuse on boys, girls and adolescents; the recognition of the importance of personality traits and competencies of the therapist in charge of this problem and the dominating role of empathy and alliance in the patient-therapist relationship.

Keywords sexual abuse, alliance, personality, psychotherapy, therapy.

\section{Personalidade e competências do psicoterapeuta de meninos, meninas e adolescentes que tiveram experiências de abuso sexual, revisão teórica

Objetivo: Compreender teoricamente a problemática do abuso sexual de meninos, meninas e adolescentes, e a necessidade de identificar as características de personalidade e competências do psicoterapeuta, devido à influencia que tem estas no estabelecimento da relação paciente- terapeuta. Método: Foi um estudo qualitativo de tipo documental descritivo, se revisaram artigos teóricos e de investigação a partir do uso de 12 bases; se realizou uma seleção das fontes de informação como critério 0 ano de publicação (2005-2013), se sistematizou a informação e se criaram categorias de análise que permitiram estabelecer e aprofundar os fatores associados ao tema do estudo. Resultados: As categorias identificadas, analisadas e teorizadas, foram: Estilo Pessoal do Terapeuta, como fator fundamental para o estabelecimento de aliança nos processos terapêuticos; Características de Personalidade do Terapeuta, componentes essenciais que intervém no estabelecimento da relação com o paciente; Abuso Sexual, problemática social que gera efeitos físicos, psicológicos e sociais para a vítima e sua família; Terapia Infantil no Abuso Sexual, processo essencial para favorecer a superação do impacto negativo do abuso; e Terapia Familiar no Abuso Sexual, processo terapêutico centrado no núcleo familiar com a rede como estratégia integradora que permite fazer ruptura com as vivencias do abuso. Conclusões: Nesta revisão teórica se identificou cinco categorias de análise essenciais para a compreensão da problemática do abuso sexual de meninos, meninas e adolescente; o reconhecimento da importância das características de personalidade e competências do terapeuta na atenção desta problemática, e a função preponderante da empatia e aliança na relação paciente- terapeuta. 


\section{ntroducción}

En la relación paciente- terapeuta es importante tener en cuenta las características de personalidad del terapeuta, ya que éstas pueden ser utilizadas para influir de alguna manera en el proceso de intervención; conocer estos aspectos puede ser un factor facilitador importante para tener mejores resultados, logrando así establecer empatía y alianzas favorecedoras de los procesos. También es necesario identificar y precisar las competencias del mismo, Tobón (2006) define el término Competencia como una conducta que se basa en el comportamiento observable, efectivo y verificable, confluyendo entonces así, el desempeño dentro de la competencia. Para el autor:

El enfoque de competencias desde lo conductual ha tenido notables desarrollos en el campo de la gestión del talento humano en las organizaciones, donde se asume con la orientación de buscar que los trabajadores posean competencias clave para que las empresas sean competitivas (p. 3).

Esta investigación parte de reconocer que en la relación paciente- terapeuta es importante la personalidad de éste, en tanto ejerce una influencia en el proceso terapéutico. La personalidad del terapeuta, puede ser un factor facilitador u obstaculizador para el logro de mejores resultados en el establecimiento de la empatía y la alianza terapéutica. Para este estudio, la personalidad es entendida como "aspectos únicos tanto internos como externos relativamente duraderos del carácter que influyen en la conducta en diversas situaciones" (Schultz \& Schultz, 2010, p. 9).

Con relación a las características deseables del terapeuta, varios autores plantean que existen tres competencias relacionales que el terapeuta debe poseer, ellas se consideran necesarias pero no suficientes, éstas son la empatía, la aceptación y la autenticidad (Beck, 1979; Rogers, 1951, citado por Ruiz \& Cano, 2002). Así mismo, Beck (1979, citado por Ruiz \& Cano, 2002), plantea que respecto a la interacción terapéutica y, desde la terapia cognitiva, son necesarios tres elementos fundamentales para mantener la relación a saber, la confianza básica, el rapport y la colaboración terapéutica.

Otros autores hablan de características personales generales para ser terapeuta (Cormier \& Cormier, 1994; Ruiz \& Villalobos, 1994, citados por Bados \& García, 2011), entra ellas se destacan el interés sincero por las personas, aceptar que hay estilos de vida diferentes, autoconocimiento, autorregulación, tener un buen ajuste psicológico general, experiencia vital, conocimiento de contextos socioculturales diversos, confianza en la propia habilidad y técnicas terapéuticas, energía y persistencia, flexibilidad, y principios éticos y profesionales.

Guy (1995), habla de motivaciones asociadas con la profesión del terapeuta, plantea unas motivaciones que son funcionales y otras que son disfuncionales. Entre las más importantes de las motivaciones funcionales están la capacidad de escuchar e interés en ello, empatía y comprensión, conocimiento y aceptación de las propias emociones positivas y negativas, tolerancia a la intimidad profunda, tolerancia a la ambigüedad o capacidad 
para soportar lo desconocido, las respuestas parciales y las respuestas incompletas. Respecto a las disfuncionales se pueden destacar: deseo de comprenderse a sí mismo y de superar los propios problemas, deseo de ayudar a otros a superar problemas no superados en la propia vida, necesidad desmesurada de dar amor o de sentirse amado, y deseo de superar la sensación de soledad mediante el contacto con otras personas.

La necesidad que se tiene frente al fenómeno del abuso sexual infantil, es identificar las características de personalidad y de competencias que debe tener el terapeuta, ésto considerando la importancia que tiene la relación terapéutica que se pueda establecer con esta población que presenta unas características particulares de vulnerabilidad y trauma. Carver y Scheier (1997) retoman a Allport quien define la personalidad como una "organización dinámica, dentro del individuo, de los sistemas psicofísicos que crean patrones característicos de la conducta, pensamientos y sentimientos" (p. 5).

Además de las definiciones de personalidad anteriormente descritas, es necesario precisar cómo a lo largo de la historia de la psicología diferentes autores como Cattell (1965) Eysenck (1953) y Asendorpf (1990, citados por Hossiep \& Paschen 2006), desarrollaron, a partir de sus teorías de la personalidad, instrumentos de evaluación para medir variables o características de la personalidad en distintas cuestiones de la vida real, calcular dimensiones que componen la personalidad, una de éstas, es la pregunta de la psicología organizacional por como elegir el mejor candidato para un determinado cargo en una empresa y como lograr determinar si éste además de contar con ciertas características de personalidad tiene las competencias necesarias para desempeñar su cargo de forma satisfactoria con eficacia y eficiencia.

Debido a que el objeto de esta investigación es precisamente el perfil de personalidad y competencias del terapeuta, es necesario definir el concepto de competencias; Tobón (2006) define el término Competencia como una conducta que se basa en el comportamiento observable, efectivo y verificable, confluyendo entonces así el desempeño dentro de la competencia.

De igual forma, es importante describir las competencias fundamentales que debe poseer un terapeuta que trabaje con realidades como el abuso sexual ya que esto permite ser más asertivos y competentes al momento de ocuparse de esta problemática.

En la investigación realizada por Jones y Morris (2007), los autores hablan del efecto, sobre el terapeuta, de trabajar con el abuso sexual, además de las características y actitudes del terapeuta en diferentes situaciones. Por esto es de interés para esta investigación evaluar estas características en el mundo laboral para elaborar un perfil de personalidad y competencias que permita a los profesionales identificar aquellas competencias que le son más útiles para abordar problemáticas como el abuso sexual infantil y así estos puedan aprovechar este conocimiento para lograr mejores resultados durante la terapia.

Cain y Seeman (2008) retoman a Rogers quien estableció que existen tres importantes características por parte del terapeuta que permitirían el desarrollo de 
cambios profundos dirigidos hacia el crecimiento del ser. Estas características son la autenticidad, aceptación incondicional positiva y empatía, características que de alguna forma hacen parte de la personalidad del terapeuta.

Riso (1996) habla de dos tipos de factores que influyen en la terapia, el primero de estos sería los factores específicos (factores técnicos relacionados con una terapia en particular) y los factores inespecíficos que incluyen: variables del paciente (personalidad, resistencia al cambio, pesimismo, motivación, fe, ganas de curarse), características del terapeuta (empatía, calidez, atención activa, sinceridad, salud mental, estilo de vida) y la relación entre ambos (alianza terapéutica, empirismo colaborativo, participación directiva, democrática, verticalista). En cualquier tipo de terapia estas variables se encuentran presentes y los resultados se encuentran influenciados por ambos factores. Se ha sugerido que mientras los factores específicos parecen explicar el $15 \%$ de la varianza de la intervención, el $45 \%$ es atribuible a los factores no específicos.

Respecto a los antecedentes investigativos, se encontró que hay pocas investigaciones sobre la personalidad del terapeuta en este tipo de contextos o realidades como el abuso sexual infantil y, las que existen se centran más en estudiar la personalidad del paciente con diferentes patologías, por esta razón se consideró importante empezar a generar cuestionamientos e investigación sobre temáticas como la enunciada anteriormente.

El tema de personalidad ha sido abordado desde diversos puntos de vista, en la mayoría de los casos la evaluación o la pregunta con respecto a ésta se ha enfocado por lo general en las patologías o en la estructura de personalidad del paciente como tal, pero muy poco se ha abordado este tema con respecto a las competencias o características que debe tener 0 desarrollar un terapeuta para abordar de manera satisfactoria las realidades de sus pacientes como en el caso del abuso sexual; sin embargo, existen investigaciones en diferentes países del mundo, las cuales de alguna manera abordan este tema sugiriendo que existen factores personales del terapeuta que intervienen de manera indudable en la relación con el paciente, las habilidades que debe tener un terapeuta con relación al tema del abuso sexual infantil deben ser: asertividad, buen sentido del humor y facilidad, para ser tanto cordial como autoritario, para trabajar en equipo, tener la capacidad de solicitar alguna consulta y segundas opiniones y, finalmente tener resistencia al fenómeno de desgastarse (Pinto 2011).

Según algunos otros autores, otras habilidades y características necesarias son el uso del lenguaje, una sana personalidad (que puede alcanzar resultados terapéuticos más satisfactorios), la capacidad de adaptación a nuevos contextos y situaciones, ser empático, tener habilidades sociales, tener conocimiento de las reglas sociales y, ser capaz de poner a un lado sus propios juicios de valor. Se resalta la importancia de realizar estudios a partir de la interacción entre terapeuta y paciente con el fin de conocer mejor las variables que influyen en el proceso terapéutico (como lo es el estilo personal del terapeuta); se considera que hay tres variables que contribuyen a la formación del estilo personal del terapeuta a saber la posición socio-profesional, la situación vital (la personalidad, la actitud y los 
posicionamientos) y, los modos dominantes de comunicación que utiliza (Castañeiras, Ledesma, García, \& Fernández, 2008; Corbella et al., 2009; Corbella, Fernández, Saúl, García \& Botella, 2008; Fernández, 1998; García \& Rodríguez, 2005; Saarnio, 2010; Santibáñez et al., 2008; Shevade, Norris, \& Swann, 2011; Topolinski \& Hertel, 2006)

En los estudios encontrados durante la investigación se halló que el terapeuta debe tener una personalidad madura, un profundo nivel de autoconocimiento y tener satisfechas sus necesidades básicas, como características esenciales para lograr mejores resultados durante el proceso terapéutico, de no ser así, el terapeuta puede no permitir un desarrollo favorable del paciente y hasta puede influir produciendo cambios negativos; se concluyó que particularidades como la flexibilidad de aprendices, la honestidad, el respeto, la honradez, la confianza, el interés, la atención, la amabilidad, la calidez y, la apertura, se asocian con una alianza mejor y, que la incertidumbre, rigidez, criticidad, distancia, tensión, aislamiento y, distracción se asocian con una alianza peor (Ackerman \& Hilsenroth 2003; Chapman 2009; Pack, 2011; Rial, Castañeiras, García, Gömez \& Fernández, 2006).
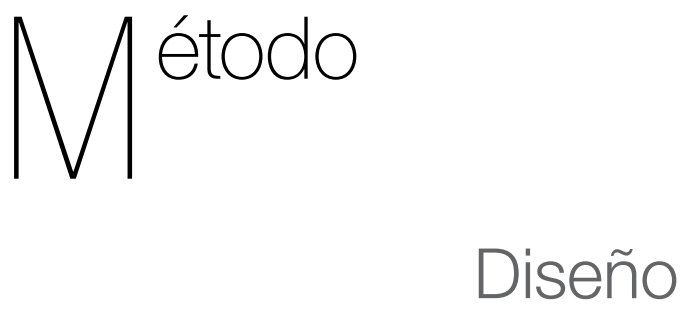

Este es un estudio cualitativo de tipo documental descriptivo.

\section{Procedimiento}

Se revisaron artículos teóricos y de investigación y de revisión teórica, utilizando para la búsqueda Bases de Datos como Ebsco, Health Source, Embase, Academic Search Complete, Fuente Académica Premier, Science Direct, Medline, MedicLatina, Scielo, Scopus, Elsevier, Scielo España, Jstor, Dialnet y, Redalyc. En un primer momento se realizó una selección de las fuentes de información en relación con los criterios del año de publicación (2005-2013), pertinencia del tema y veracidad de la información; se tuvieron en cuenta términos de búsqueda como: Abuso sexual, Violencia sexual infantil, personalidad, competencias del terapeuta, terapia, psicoterapia, entre otros. Posterior a la lectura y sistematización de la información, se crearon las categorías de análisis que permitieron abordar y profundizar en factores asociados al tema de interés.

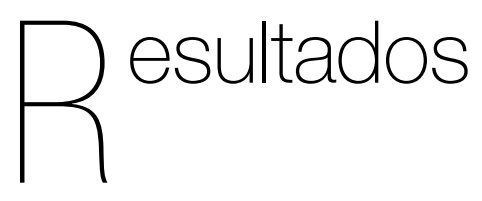

A partir de la revisión de las investigaciones seleccionadas acerca del tema de interés, se relacionaron las siguientes categorías: Estilo Personal del Terapeuta, Características de Personalidad del Terapeuta, Abuso Sexual, Terapia Infantil en el Abuso Sexual y Terapia Familiar en el Abuso Sexual; las cuales permitieron identificar aspectos relacionados con el tema de interés. 


\section{Estilo Personal del Terapeuta}

En el ejercicio de la psicoterapia en problemáticas como el abuso sexual de niños, niñas y adolescentes, se requiere por parte del terapeuta bases sólidas, que permitan un oportuno y pertinente acompañamiento. En este punto, juega un papel fundamental el estilo personal del terapeuta, ya que lograr una efectiva alianza terapéutica y esperar que los factores externos y propios del consultante jueguen favorablemente, es el ejercicio común que ha de tenerse en cuenta si se quiere lograr un proceso confiable y con resultados positivos tanto para el consultante como para el terapeuta (Rondón, Otálora \& Salamanca, 2009).

Los estudios sobre el proceso terapéutico en la última década, han resaltado en la práctica profesional de la psicoterapia no sólo la presencia de técnicas articuladas a teorías específicas, sino también al estilo personal del terapeuta, el cual tiene un papel fundamental en los procesos de acompañamiento terapéutico a pacientes con diversas patologías (Beutler et al., 2004; Corbella \& Botella, 2004; Rial et al., 2006).

Corbella y Fernández (2006) y Corbella et al. (2008) definen el estilo personal del terapeuta como el conjunto de condiciones particulares del terapeuta que lo llevan a actuar de un modo específico en su tarea. Para Fernández y García (1998), el estilo se refiere a las características usuales que cada terapeuta aplica en su quehacer profesional, que resultan de su manera de ser y su personalidad. El estilo personal del terapeuta, su papel en los procesos terapéuticos y sus efectos en la psicoterapia, resultan ser de interés para algunos estudios del medio. Entendido como un constructo multidimensional esencial en el proceso terapéutico, integra las habilidades, rasgos y actitudes del terapeuta y el cual influye en los alcances y resultados de los procesos terapéuticos (Fernández \& García, 1998; Fernández, García, Lo Bianco \& Corbella-Santomá, 2003). Este constructo resulta ser considerado por Barón y Kenny (1986, como se cita en Castañeiras et al., 2008) como regulador de la terapia, que repercute sobre los efectos de las acciones terapéuticas, efectos que varían dependiendo de las características del paciente, el motivo de consulta, la patología y el escenario.

Fernández et al. (2003), señalan que el estilo personal del terapeuta es una variable dimensional con permanencia en el tiempo, aunque dinámica debido al entrenamiento, etapa del ciclo de vida y condiciones laborales del terapeuta. Igualmente, el estilo del terapeuta es previsto y modificado por aspectos propios de la base teórica de la psicoterapia y la experiencia en el ejercicio profesional (Castañeiras, García, Lo Bianco \& Fernández, 2006; Fernández, García, Lo Bianco \& Corbella-Santomá, 2000). En el estudio realizado por Rial et al. (2006) se nombran como los principales desarrollos teóricos respecto al estilo terapeuta aquellos que han permitido diferenciar entre variables de rasgo (observable e inferido) y variables de estado (estados: observables e inferidos). En los primeros, se destacan el entrenamiento del terapeuta, la experiencia, estilo interpersonal.

Las investigaciones del estilo del terapeuta, se han enmarcado en tres líneas principalmente, la primera se orienta a la conceptualización y la evaluación del 
estilo personal del terapeuta (Fernández et al., 2003; Fernández, Gómez, Castañeiras \& Rial, 2005); la segunda, dirigida a la investigación aplicada en diversos escenarios y campos (Corbella-Santomá, 2002; Malik et al., 2002; Popp, Goncalvez, \& Caspar, 2004; Rial et al., 2006; Vega, 2006 ); y la tercera, se centra en el estilo terapéutico como recurso formativo en los programas de entrenamiento en psicoterapia, enmarcadas en la adquisición de competencias por parte del terapeuta, que posibiliten el ajuste de ese estilo (Beutler et al., 2004; Fernández, 2001; Hill \& O’ Brien, 1999).

\section{Características de Personalidad del Terapeuta}

Respecto a esta categoría de análisis se encontró en la pesquisa realizada, que existen características de personalidad del terapeuta que pueden facilitar o entorpecer un proceso de terapia en los diversos contextos donde éstos se dan.

El estudio realizado por Saarnio (2010) cuyo objetivo fue investigar si se producen diferencias de género en la personalidad, características o funcionamiento interpersonal entre los terapeutas de pacientes con abuso de sustancias; mostró en sus resultados que los terapeutas mujeres obtuvieron puntuaciones más altas que los terapeutas varones en los factores de la personalidad de Amabilidad y Apertura a la experiencia; las mujeres también fueron mejores que los hombres en el funcionamiento interpersonal en todas las dimensiones de la misma como empatía, autenticidad y, respeto de los clientes.
Rogers citado por Cain y Seeman (2008), estableció la autenticidad, la aceptación incondicional positiva y la empatía como características esenciales que debe poseer cada terapeuta, con el fin de desarrollar cambios profundos dirigidos hacia el crecimiento del ser. Según Pinto (2011), existen factores personales del terapeuta que intervienen de manera indudable en la relación con el paciente, las habilidades que debe tener un terapeuta con relación al tema del abuso sexual infantil deben ser la asertividad, buen sentido del humor y facilidad, para ser tanto cordial como autoritario, para trabajar en equipo, tener la capacidad de solicitar alguna consulta y segundas opiniones y, finalmente tener resistencia al fenómeno de desgastarse.

Para otros autores, otras habilidades y características necesarias son el uso del lenguaje, una sana personalidad (que puede alcanzar resultados terapéuticos más satisfactorios), la capacidad de adaptación a nuevos contextos y situaciones, ser empático, tener habilidades sociales, tener conocimiento de las reglas sociales y, ser capaz de poner a un lado sus propios juicios de valor. Se resalta la importancia de realizar estudios a partir de la interacción entre terapeuta y paciente con el fin de conocer mejor las variables que influyen en el proceso terapéutico (como lo es el estilo personal del terapeuta); se considera que hay tres variables que contribuyen a la formación del estilo personal del terapeuta a saber: la posición socioprofesional, la situación vital (la personalidad, la actitud y los posicionamientos) y, los modos dominantes de comunicación que utiliza (Castañeiras et al., 2008; Corbella et al., 2009; Corbella et al., 2008; Fernández, 1998; García \& Rodríguez 2005; Topolinski \& Hertel, 2007; Saarnio, 
2010; Santibáñez et al., 2008; Shevade, Norris \& Swann, 2011)

Se encontró también que el terapeuta debe tener una personalidad madura, un profundo nivel de autoconocimiento y tener satisfechas sus necesidades básicas, como características esenciales para lograr mejores resultados durante el proceso terapéutico, de no ser así, el terapeuta puede no permitir un desarrollo favorable del paciente y hasta puede influir produciendo cambios negativos; se concluyó que particularidades como la flexibilidad de aprendices, la honestidad, el respeto, la honradez, la confianza, el interés, la atención, la amabilidad, la calidez $y$, la apertura, se asocian con una alianza mejor y, que la incertidumbre, rigidez, criticidad, distancia, tensión, aislamiento y, distracción se asocian con una alianza peor (Ackerman \& Hilsenroth 2003; Chapman 2009; Pack, 2011; Rial et al., 2006).

\section{Abuso Sexual}

El abuso sexual infantil es definido como cualquier conducta sexual sostenida entre un adulto y un menor, donde el factor determinante es la ausencia de consentimiento (Echeburúa \& Corral, 2006). Para Acero (2009), en algunos casos los menores resultan ser abusados por otros menores, que pueden ejercer algún poder. El abuso puede suponer un contacto físico o la utilización del menor como objeto de estimulación sexual. De acuerdo el estudio de Pereda, Guilera, Forns y Gómez (2009), el cual se centró en la revisión de artículos de 22 países, al menos el $7.9 \%$ de hombres y el $19.7 \%$ de mujeres antes de los 18 años han sufrido al tipo de abuso sexual. Podesta y Rovea
(2003), señalan que las niñas son más susceptibles a ser abusadas, y esta sufre va en aumento cuando son criadas por padrastros.

El abuso sexual infantil resulta ser una problemática actual, que ha demandado de diversas disciplinas con el propósito de comprender más este fenómeno y aportar a su disminución y tratamiento, la tasa de abuso es cada vez mayor y se orienta a casos de abuso sexual grave, donde existe presencia de contacto físico, actos respetivos y con consecuencias negativas en el desarrollo emocional del menor (Cantón \& Justicia, 2008; Echeburúa \& Guerricaechevarría, 2011; Pereda et al., 2009). Showalter (2007) señala que el abuso sexual resulta ser una problemática social significativa, que presenta consecuencias tanto para las víctimas como para las familias, y que se ha convertido en un costo para la sociedad.

Mebarak, Martínez, Sánchez, y Lozano (2010) hallan en su estudio los siguientes factores de riego del abuso sexual: "sexo femenino, tener entre 11 y 15 años, padecer insuficiencias económicas y culturales, haber sufrido previamente otros tipos de maltrato infantil y haber desarrollado una baja autoestima, sumisión a la autoridad y poca confianza en los adultos" ( $p$. 134). Entre los factores de riesgo también figura las relaciones distantes entre los padres, la poca confianza, consumo de sustancia psicoactivas y la guerra (Lee \& Lathrop, 2010; Romero, 2010, Serrano, Pérez, \& Chaskel, 2010; Usta \& Farver, 2010; Western, 2006). Cada uno de estos factores, según los autores actúan como causantes y mantenedores del abuso.

Respecto a las consecuencias de las víctimas, estas se orientan a alterar 
principalmente el funcionamiento psicológico, especialmente cuando el agresor es un miembro de la familia. A largo plazo varían las consecuencias, aunque se ha identificado que niño y niñas abusadas presentan a futuro alteraciones emocionales o de comportamientos sexuales. Los estudios concuerdan que ante el apoyo familiar y el acampamiento psicológico el impacto negativo del abuso es menor (Cantón, Cantón, Justicia, \& Cortés, 2011; Echeburúa \& Corral, 2006; Pereda, Gallardo, \& Jiménez Padilla, 2011).

Para Finkelhor (2008) los efectos del abuso sexual concuerdan los de otro tipo de agresiones, de ahí, que los diferentes tipos de victimización presenten síntomas similares. Para el autor, la diferencia se encuentra en las conductas sexuales inapropiadas que pueden presentarse en las víctimas de abuso abusados.

\section{Terapia Infantil en el Abuso Sexual}

Estudios sobre abuso sexual concuerdan en concluir que los niños y niñas que han sido objeto de abuso sexual manifiestan alteraciones psicológica, física y comportamentales; entre estas manifestaciones se resaltan: cambios en el estado de ánimo, sentimientos ambivalentes, pesadillas, retraimiento, agresividad, cambios en la alimentación, cambios en las relaciones familiares, y sentimientos de inadecuación sexual. Estas manifestaciones de acuerdo a Sánchez (2002), Podestá y Laura (2005) y Echeburrúa y Guerrivaechevarría (2000) resaltan la necesidad directa que existe de acompañar mediante procesos terapéuticos estas vivencias. En los casos de abuso sexual infantil es fundamental la intervención terapéutica, de carácter interdisciplinar. Para el alcance de las metas, el autor menciona tres modalidades de atención: individual, grupal y familiar, estas se deben orientar por medio de la utilización de técnicas de entrenamiento en relajación, desensibilización y visualización Rodríguez (2003).

La importancia del carácter interdisciplinar también lo resaltan Assis, Palomino y Rosselli (2009); y Bernik, Laranjeiras y Corregiari (2003), quienes alertan de la necesidad de un trabajo combinado para las víctimas de abuso sexual, más aun cuando son diagnosticadas con Trastorno de Estrés Postraumático. Por la complejidad de las manifestaciones sintomáticas que presentan los niños y niñas víctimas de abuso sexual, Vallejo y Terranova (2009) recomiendan la combinación de técnicas entre las que resaltan la relajación, la reestructuración cognitiva, la psicoterapia psicodinámica y el uso de psicofármacos. El tratamiento a los casos de abuso sexual, depende del tipo de criterio con que se enfoque la intervención, según Intebi (2008) este puede enfocarse en protección infantil, en los aspectos legales o en los aspectos clínicos.

En los estudios de Echeburúa y Guerricaechevarría (2000, como se cita en Echeburúa \& Guerricaechevarría, 2002; 2011) respecto a la terapia en víctimas de abuso, manifiestan que no necesariamente una intervención clínica es una variable de obligatoriedad para el apoyo, en diversos casos "el apoyo familiar, las relaciones sociales y la reanudación de la vida cotidiana son suficientes como factor de protección al menor" (Echeburúa \& Guerricaechevarría, 2011, p. 471). Las victimas que hacen parte de los tratamientos 
psicológicos, presentan síntomas psicopatológicos intensos, entre los que se pueden destacar inadaptación, ansiedad, depresión, o alteraciones.

El rol del terapeuta puede estar centrado en la orientación, asesoría y apoyo a la familia, a la evaluación del desarrollo psicológico del menor y a un tratamiento, en cada uno de estos momentos, el terapeuta debe identificar el momento oportuno de la misma y la generación de acompañamiento acorde a la edad del menor y las necesidades específicas (Echeburúa \& Guerricaechevarría, 2000; Echeburúa, Guerricaechevarría \& Amor, 2002; Horno, Santos \& Molino, 2001; Pack, 2011; Shevade, Norris \& Swann, 2011).

Aunque el menor es el eje central del acompañamiento, la intervención psicológica con los familiares es indispensable., como mencionan Echeburúa y Guerricaechevarría (2011). "Ellos van a tener que afrontar una situación dolorosa, así como todas las circunstancias que se deriven de la revelación de los abusos, y son los que deben garantizar la protección y seguridad del menor" (p. 471). En este caso, el terapeuta debe orientar la intervención a que los familiares y cuidadores aprendan a supervisar la evolución del menor, generar estrategias para brindarle seguridad y enseñarle estrategias de afrontamiento favorables (Echeburúa \& Guerricaechevarría, 2000).

\section{Terapia Familiar en el Abuso Sexual}

Los estudios del abuso sexual en niños, niñas y adolescentes cuenta con una tradición respecto a las consecuencias del abuso sobre el desarrollo integral, pero resultan ser pocos los estudios que se han centrado en el impacto que tiene este evento sobre la familia, y las formas terapéuticas para acompañar este escenario social (Jaén \& Garrido, 2009).

Para Cirilo y Di Blasco (1991) y Noguerol (1995), las variables que concurren para establecer las necesidades de la familia en los procesos de afrontamiento de las situaciones de abuso de los menores son múltiples y muy variadas, entre estas variables figuran: duración en el tiempo, frecuencia, tipo de prácticas sexuales, relación con el abusador, actitud de la familia, etapa evolutiva del menor y el ciclo vital familiar, los efectos sociales y contextuales del abuso -procesos y efectos judiciales-.

Los procesos terapéuticos familiares, deben contemplar no solo estas variables, sino las lógicas y dinámicas familiares que subyacen al interior de estas familias, esto debido a que en la práctica los modelos de constructos familiares son diversos (Feixas \& Villegas, 1993), además, de acuerdo a Ugazio (2001) y Linares, (2002) a estas familias subyace patrones de interacción rígidos y simétricos que dominan las relaciones paterno filiales en el caso del abuso sexual.

Jaén y Garrido (2009) expresan lo evidente que resulta ser en los menores la incapacidad para asignar significado a la experiencia de abuso, de ahí, que resulte fundamental:

La respuesta del contexto en su interacción con el menor traumatizado y fundamentalmente de aquellas figuras emocionalmente vinculantes, puede tener un efecto reparador en 
cuanto a que pueden facilitar un discurso en torno a experiencia traumática aportando tonalidades emocionales y significados que permiten al menor salir de un ciclo ininterrumpido en el que las emociones y las conductas están carentes de significados o en el peor de los casos están asociados a construcciones del sí-mismo patologizantes y desadaptativas (p.335).

Es en este punto donde se resalta la pertinencia de la terapia familiar, la cual no resulta excluyente con el trabajo individual (Linares 2002; Perrone, 1998; Ugazio, 2001). Esta se centra en crear situaciones en que los familiares comprometan emocionalmente la situación vivida por el menor y por ellos mismos (Friedlander, Escudero, \& Heatherington, 2009; Garrido \& Espina, 1995), esto posibilita que las familias adopten nuevas posiciones relacionales y recuperen la esperanza de cambio. Igualmente, resulta importante que:

La intervención con otros agentes sociales se pueda hacer teniendo en cuenta que el foco es el menor y el significado que éste se atribuye a sí mismo y a su entorno tras la experiencia traumática, y su incapacidad para darle sentido (Jaén \& Garrido, 2009, p. 336).

Esta revisión realizada a diversas investigaciones desarrolladas en torno al abuso sexual y su abordaje terapéuti$\mathrm{co}$, permiten identificar las categorías de análisis: Estilo Personal del Terapeuta, Características de Personalidad del Terapeuta, Abuso Sexual, Terapia Infantil en el Abuso Sexual y Terapia Familiar en el Abuso Sexual. Estas categorías desarrolladas individualmente, presentan de base una articulación entre sí, la cual no debe ser desconocida por parte de los profesionales que de una $\mathrm{u}$ otra manera atienden y estudian esta población, y que resulta ser el hallazgo principal de este estudio: La manera como se presentan y se articulan estas categorías evidencian estructuras personales del terapeuta que son favorecedoras de la relación paciente-terapeuta y los avances significativos del acompañamiento terapéutico; o por el contrario, estas estructuras del terapeuta pueden ser obstaculizadoras de la alianza y el progreso del paciente víctima de abuso sexual.

Estas categorías proponen la base fundamental a tener presente en la atención a la población infantil y adolescente víctima del abuso sexual: el ser del terapeuta como pilar del proceso, dado que el estilo y las características de personalidad del terapeuta influyen en la terapia individual y familiar, y en la posibilidad dar solución a las secuelas emocionales, sociales, cognitivas y familiares propias de las víctimas del abuso sexual.

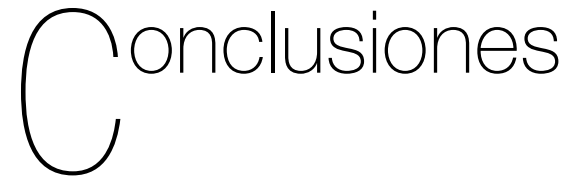

La revisión teórica realizada en este estudio, permitió identificar categorías de análisis que ayudan a la comprensión de la problemática del abuso sexual de niños, niñas y adolescentes, más específicamente, la importancia de las características de personalidad 
y competencias del terapeuta, y la relación de estas con el establecimiento de empatía y alianza en la relación paciente- terapeuta.

Respecto a las categorías de análisis identificadas y teorizadas, se puede concluir que el Estilo Personal del Terapeuta tiene un papel primordial en el alcance de una efectiva alianza terapéutica, el cual se caracteriza por permanecer en el tiempo, ser susceptible entrenamiento, transformarse a lo largo de ciclo de vital y modificarse por aspectos propios de la base teórica de la psicoterapia. El terapeuta debe estar atento a este, para favorecer un proceso confiable y efectivo en el proceso terapéutico de niños, niñas y adolescentes con vivencias de abuso sexual, y con ello al establecimiento de una relación favorable y positiva entre el paciente y el terapeuta.

De igual forma, las características de Personalidad del Terapeuta intervienen directa e indirectamente en el establecimiento y consolidación de la relación con el paciente. Entre los factores identificados que juegan un papel esencial en el proceso terapéutico de niños, niñas y adolescentes víctimas de abuso sexual figuran: la asertividad, alto nivel de autoconocimiento, el buen sentido del humor, el trabajo grupal y colaborativo, el establecimiento de límites de autoridad articulados a espacios abiertos de diálogo, la capacidad de adaptación a nuevos contextos, y la presencia de repertorios y destrezas sociales.

El Abuso Sexual es una problemática social significativa, la cual genera consecuencias personales, familiares y sociales en las victimas. Entre los efectos presentes se encuentran: alteraciones emocionales o de comportamientos sexuales, tales como: cambios en el estado de ánimo, sentimientos ambivalentes, pesadillas, retraimiento, agresividad, alteración en la alimentación, cambios en las relaciones familiares, y sentimientos de inadecuación sexual.

Con relaciona a la terapia Infantil en el Abuso Sexual, los estudios concuerdan que ante el apoyo familiar y el acompañamiento psicológico son esenciales para que el niño, la niña y el adolescente supere el impacto negativo del abuso. El terapeuta debe tener presente en este proceso, las interacciones sociales y las actividades cotidianas como ejes centrales de adecuación por medio de la intervención.

Finalmente, en la Terapia Familiar en el Abuso Sexual los procesos terapéuticas con la red de apoyo familiar, se presentan como acciones de gran efecto reparador, debido a que estas figuras afectivas para el niño, la niña y el adolescente aportan seguridad emocional que permite hacer ruptura con las vivencias del abuso. Este proceso terapéutico, ha de centrase en comprometer a los familiares emocionalmente en la situación experimentada por el niño, la niña o el adolescente, esto debido a que los familiares ante la vivencia de abuso se ven igualmente afectados, lo que genera en ellos choques emocionales que bloquean en formas positivas y adecuadas de apoyar al niño y al adolescente. Un terapeuta atento a esto, y con estructuras claras en su estilo pueden movilizar procesos en las familias que aportan sustancialmente al proceso terapéutico de la víctima de abuso sexual. 


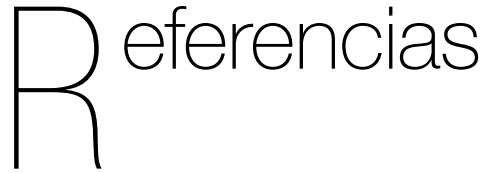

Acero, A. (2009). Informes periciales sexológicos. Bogotá: Instituto Nacional de Medicina legal y ciencias forenses.

Ackerman, S., \& Hilsenroth, M. (2003). A review of therapist characteristics and techniques positively impacting the therapeutic alliance. Clinical Psychology Review, 23, 1-33.

Assis, C., Palomino, V., \& Rosselli, M. (2009). Mulheres vitimadas sexualmente - perfil sociodemográfico e analise do atentimiento em um entro de referencia. Ciencia \& Saudé, 27 (1), 22-27.

Bados, A., \& García, E. (2011). Habilidades Terapéuticas. Departamento de Personalidad, Evaluación y Tratamiento Psicológicos, Facultad de Psicología, Universidad de Barcelona. Recuperado de: $\quad$ http://diposit.ub.edu/dspace/ bitstream/2445/18382/1/Habilidades\%20 terap\%C3\%A9uticas.pdf

Bernik, M., Laranjeiras, M., \& Corregiari, F. (2003). Tratamiento farmacológico do trastorno de estresse pos-traumático. Revista Brasileira de Psiquiatria, 25, 46-50.

Beutler, L., Malik, M. Alimohamed, S., Harwood, T., Talebi, H., Noble, S., \& Wong, E. (2004). Therapist variables. En: M.J.Lambert (Eds.). Bergin and Garfiueld' $s$ Handbook of Psychotherapy and Behavior Change (Fifth Edition) (pp. 227306). New York: John Wiley.
Cain, D., \& Seeman, J. (2008). Humanistic Psychotherapies: Handbook of Research and Practice. Washington: American Psychiatry Association.

Cantón, D., \& Justicia, F. (2008). Afrontamiento del abuso sexual infantil y ajuste psicológico a largo plazo. Psicothema, 20, 509-515.

Cantón, D., Cantón, J., Justicia, F., \& Cortés, M. (2011). Un modelo de los efectos del abuso sexual infantil sobre el estrés postraumático: el rol mediador de las atribuciones de culpa y afrontamiento de evitación. Psicothema, 23, 66-73.

Carver, C., \& Scheier, M. (1997). Teorías de la Personalidad (3ra Ed). México, D.F: Prentice Hall Hispanoamericana.

Castañeiras, C., Ledesma, R., García, F., \& Fernández, H. (2008). Evaluación del Estilo Personal del Terapeuta: Presentación de una Versión Abreviada del Cuestionario EPT-C. Terapia Psicológica, 26(1), 5-13.

Castañeiras, C, García, F., Lo Bianco, J., \& Fernández, H. (2006). Modulating Effect of Experience and Theoretical-Technical Orientation on the Personal Style of the Therapist. Psychotherapy Research, 16(5), 587-593.

Chapman, B. (2009). Personality Traits and the working alliance in psychotherapy trainees: an organizing role for five factor model. Jounal of social \& clinical psychology, 28(5), 577-596.

Cirilo, S., \& Di Blasco, P. (1991). Niños maltratados (Diagnostico y terapia familiar). Barcelona: Paidós 
Corbella, S., \& Botella, L. (2004). Investigación en Psicoterapia: Proceso, Resultado y Factores Comunes. Madrid: Vision Net.

Corbella, S., \& Fernández, H. (2006). El terapeuta, posiciones, actitudes y estilos personales. En L. Botella (Ed.), Construcciones, narrativas y relaciones: Aportaciones constructivistas y construccionistas a la psicoterapia. Barcelona: Edebe.

Corbella, S., Balmaña, N., Fernández, H., Angel, L., Botella, L., \& García, F. (2009). Estilo Personal del Terapeuta y Teoría de la Mente. Revista Argentina de Clínica Psicológica,18(2), 125-133.

Corbella, S., Fernández, H., Saúl, L. A., García, F., \& Botella, L. (2008). Estilo personal del psicoterapeuta y dirección de intereses. Apuntes de Psicología, 26(2), 281-289.

Corbella, S. (2002). Compatibilidad entre el estilo personal del terapeuta y el perfil personal del paciente. Tesis doctoral no publicada. Universidad Ramón Llull. España.

Echeburúa, E., \& Corral, P. (2006). Secuelas emocionales en víctimas de abuso sexual en la infancia. Cuadernos de Medicina Forense, 12, 75-82.

Echeburúa, E., \& Guerricaechevarría, C. (2000). Abuso sexual en la infancia: víctimas y agresores. Barcelona. Ariel.

Echeburúa, E., \& Guerricaechevarría, C. (2011). Tratamiento Psicológico De Las Víctimas De Abuso Sexual Infantil Intrafamiliar: Un Enfoque Integrador. Behavioral Psychology, 19(2), 469-486.
Echeburúa, E., Guerricaechevarría, C., \&Amor, P.J. (2002). Indicaciones terapéuticas para los menores víctimas de abuso sexual. En M. Lameiras (dir.), Abusos sexuales en la infancia. Abordaje psicológico y jurídico (pp. 115-137). Madrid: Biblioteca Nueva.

Feixas, G., \& Villegas, M. (1993). Constructivismo y psicoterapia. Barcelona: PPU.

Fernández, H. (1998). El estilo personal del psicoterapeuta. En Oblitas Guadalupe, L. A. Quince enfoques terapéuticos contemporáneos. México, D.F.: El Manual Moderno.

Fernández, H., \& García, F. (1998). El estilo personal del terapeuta: Inventario para su evaluación. En: S. Gril, A. Ibáñez, I. Mosca y P.L.R. Sousa (Eds.), Investigación en Psicoterapia (pp. 76-84). Pelotas: Educat.

Fernández, H. (2001). Formación de terapeutas. Entrenamiento en habilidades terapéuticas. En: H. Mesones Arroyo (Ed.). Curso de Psicoterapia IV: La formación del psicoterapeuta (pp. 75-90). Buenos Aires: Ananke.

Fernández, H., García, F., Lo Bianco, J. \& Corbella-Santomá, S. (2003). Assessment Questionnaire on The Personal Style of the Therapist PST-Q. Clinical Psychology and Psychotherapy, 10, 116-125.

Fernández, H., García, F., Lo Bianco, J. \& Corbella-Santomá, S. (2000, septiembre). Estilos personales e terapeutas psicoanalíticos, cognitivos e integrativos. Comunicación presentada en el 2do Congresso Latinamericano de Pesquisa em Psicoanalise e Psicoterapia. Gramado, Brasil. 
Fernández, H. Gómez, B. Castañeiras, C. \& Rial, V. (2005). Normalization of the Assessment Questionnaire of the Personal Style of the Therapist (PST-Q) in apopulation of Argentinian Psychotherapists. Comunicación presentada en el 36th Annual Meeting of the Society for Psychotherapy Research. Quebec, Canadá.

Finkelhor, D. (2008). Efectos (Victimología infantil). En J. Sanmartín (Ed.), Violencia contra niños (4 ${ }^{\mathrm{a}}$ ed., pp. 179-202). Barcelona: Ariel.

Friedlander, M., Escudero, V., \& Heatherington, L. (2009) La alianza terapéutica en la terapia familiar y de pareja. Barcelona: Paidós.

García, A., \& Rodríguez, J. C. (2005). Factores Personales en la Relación Terapéutica. Revista de la Asociación Española de Neuropsiquiatría 25(96) 29-36.

Garrido, M., \& Espina, A. (1995). Terapia Familiar. Aportaciones psicoanalíticas y transgeneracionales. Madrid: Fundamentos.

Guy, J. (1995). La vida personal del psicoterapeuta: el impacto de la práctica clínica en las emociones y vivencias del terapeuta. Buenos Aires: Paidós.

Hill, C., \& O'Brien, K. (1999). Helping skills: facilitating exploration, insight, and action. Washington D.C.: American Psychological Association.

Horno, P., Santos, A., \& Molino, C. (2001). Abuso sexual infantil: manual de formación para profesionales. Madrid: Save the Children España.
Hossiep, R., \& Paschen, M. (2006). Manual Bochum de Personalidad y Competencias. Adaptación al español de Arribas, D., Corral, S. \& Peña, J. (2006): Madrid: TEA Ediciones.

Intebi, I. (2008). Valoración de las sospechas de abuso sexual infantil. Colección Documentos Técnicos. Dirección General de Políticas Sociales, Consejería de Empleo y Bienestar Social, Gobierno de Cantabria, España.

Jaén, P., \& Garrido, M. (2009). Psicoterapia familiar en casos de abuso sexual: la utilización de las emociones como recurso para el restablecimiento de límites en los trastornos de la conducta antisocial. Apuntes de Psicología, 27, (23), 321-338.

Jones, F., \& Morris, M. (2007). Working with child sexual abuse: a systemic perspective on whether children need to tell their therapist details of the abuse for healing to take place. Journal of Family Therapy, 29, 222-237.

Lee, C., \& Lathrop, S. (2010). Child abuserelated homicides in New Mexico: A 6-year retrospective review. Journal of Forensic Sciences, 55 (1), 100-103.

Linares, J. (2002). Del abuso y otros desmanes: el maltrato familiar, entre la terapia y el control. Barcelona: Paidós.

Malik, M., Fleming, J., Pénela, V., Harwood, M., Best, S., \& Beutler, L. (2002). Which therapist for which client? The interaction of therapist variation and client characteristics in affecting rates of change: An effectiveness study. Comunication presentada en el 33th Annual Meeting 
of Society for Psychotherapy Research. Santa Bárbara, USA.

Mebarak, M., Martinez, M., Sanchez, A., \& Lozano, J. (2010). Una revisión acerca de la sintomatología. Psicología desde el Caribe, 25, 128-154.

Noguerol, V. (1995). Implicaciones del sistema legal en el abuso sexual infantil: un saco lleno de preguntas, dilemas y frustraciones. Bienestar y Protección Infantil, 4, 76-87.

Pack, M. (2011). Discovering an integrated framework for practice: a qualitative investigation of theories used by social workers working as sexual abuse therapists. Journal of Social Work Practice, 25(1), 79-93.

Pereda, N., Guilera, G., Forns, M., \& Gómez, J. (2009). The prevalence of child sexual abuse in community and students samples: A meta-analysis. Clinical Psycholology Review, 29, 328-338.

Pereda, N., Gallardo \& Jiménez, R. (2011). Trastornos de personalidad en víctimas de abuso sexual infantil. Actas Españolas de Psiquiatría, 39, 131-139.

Perrone, R. (1998). Violencia y abusos sexuales en la familia: un abordaje sistémico y comunicacional. Barcelona: Paidós.

Pinto, C. (2011). Aproximación a la psicoterapia con niños, niñas y adolescentes sobrevivientes de abuso sexual. Revista Electrónica de Psicología social POIÉSIS, 211-15.

Podestá, M., \& Rovea, O. (2003). Abuso Sexual infantil intrafamilar. Un abordaje desde el Trabajo Social. Ed. Espacio.
Podestá, M., \& Laura, R. (2005). Abuso Sexual Infantil Intrafamiliar, Un abordaje desde el Trabajo Social. Buenos Aires: Espacio.

Popp, M., Goncalves, M., \& Caspar, F. (2004, junio). Thepsychotherapists' attention in the course of an intake interview. A comparison between experts and novices. Comunicación presentada en el 35th Annual Meeting of Society for Psychotherapy Research. Roma, Italia.

Rial, V., Castañeiras, C., García, F., Gómez, B., \& Fernández, H. (2006). Estilo personal de terapeutas que trabajan con pacientes severamente perturbados: un estudio cuantitativo y cualitativo. Revista de la Asociación Española de Neuropsiquiatría 26(98), 191-208.

Riso, W. (1996). La Terapia cognitivo informacional: Crítica a las terapias tradicionales e implicaciones clínicas. Medellín: Editorial.

Rodríguez, A. (2003). Intervención interdisciplinaria en casos de abuso sexual infantil. Universitas Psychologica, 2(1), 57-60.

Rondón, B., Otálora, B., \& Salamanca C. (2009). Factores que influyen en la deserción terapéutica de los consultantes de un centro universitario de atención psicológica. International Journal of Psychological Research, 2(2), 137-147.

Romero, V. (2010). Parental substance abuse and child neglect: A controlled trial of a developed treatment manual. Romero, Valeria I: U Nevada, Las Vegas, US.

Ruiz, J., \& Cano, J. (2002). Manual de psicoterapia cognitiva. R\&C Editores. Disponible en: http://www. 
psicologia-online.com/ESMUbeda/ Libros/Manual/manual7.htm

Saarnio, P. (2010). Big Five Personality Traits and Interpersonal Functioning in Female and Male Substance Abuse Therapists. Substance Use \& Misuse, 45(10), 14631473.

Sánchez, R. (2002). Abuso sexual aspectos teóricos. México, D.F.: Solar.

Santibáñez, P. Román, M. Chenevard, C. Espinosa, A. Irribara, D., \& Müller, P. (2008). Variables Inespecíficas en Psicoterapia. Terapia Psicológica, 26 (1) 89-98.

Schultz, D., \& Schultz, E. (2010) Teorías de la personalidad (9na Ed). México, D.F.: Cengage Learning.

Serrano, A., Pérez, P., \& Chaskel, R. (2010). Abuso sexual, reconocimiento y manejo. CCAP, 8(3), 39-40.

Shevade, D., Norris, E. \& Swann, R. (2011). An Exploration of Therapists' Reactions to Working with Children Displaying Sexually Problematic Behaviour: A Thematic Analytic Study. Journal of Child Psychotherapy, 37 (1), 52-74.

Showalter, L. (2007). The magic circle: $A$ personal safety curriculum for kindergarten through third grades. [Unpublished M.S.W.], California State University, Lo Beach, United States - California.
Tobón, S. (2006). Aspectos básicos de la formación basada en competencias. Recuperado el 21 de Octubre de 2010 en: http://maristas.org.mx/gestion/web/ doctos/aspectos_basicos_formacion_ competencias.pdf

Topolinski, S., \& Hertel, G. (2007). The role of personality in psychotherapists' careers: Relationships between personality traits, therapeutic schools, and job satisfaction. Psychotherapy Research, 17(3), 378-390.

Ugazio, V. (2001). Historias permitidas, historias prohibidas. Barcelona: Paidós.

Usta, J., \& Farver, J. (2010). Child sexual abuse in Lebanon during war and peace. Child Care, Health \& Development, 36(3), 361-368.

Vallejo, Á., \& Terranova, L. (2009). Estrés postraumático y psicoterapia de grupo en militares. Terapia Psicológica, 27(1), 103-112.

Vega, E. (2006). El psicoterapeuta en neonatología: rol y estilo personal. Buenos Aires: Lugar Editorial.

Western, G. (2006). The relationship of psychotic disorders, childhood sexual abuse and psychosocial phenomena. [Unpublished Psy.D.], Alliant International University, San Diego, United States California 